\title{
A Hybrid Approach to Failure Analysis Using Stochastic Petri Nets and Ranking Generalized Fuzzy Numbers
}

\author{
Abolfazl Doostparast Torshizi and Jamshid Parvizian \\ Department of Industrial Engineering, Isfahan University of Technology, Isfahan 84156-83111, Iran \\ Correspondence should be addressed to Abolfazl Doostparast Torshizi, a.doostparast@aut.ac.ir \\ Received 25 April 2012; Accepted 4 September 2012 \\ Academic Editor: Zeng-Guang Hou
}

Copyright ( 2012 A. Doostparast Torshizi and J. Parvizian. This is an open access article distributed under the Creative Commons Attribution License, which permits unrestricted use, distribution, and reproduction in any medium, provided the original work is properly cited.

\begin{abstract}
We present a novel failure analysis approach combining structural properties of stochastic Petri Nets and flexibility of fuzzy logic. Firstly, we develop a powerful fuzzy ranking technique. We analyze major drawbacks of existing ranking techniques. Then we demonstrate the capabilities of the presented algorithm to overcome such drawbacks. The approach considers weight, spread, and difference of $x$ coordinate of the center of gravity (COG) point of each fuzzy number and is able to deal with a wide variety of fuzzy numbers. Using this technique, we utilize isomorphism between stochastic Petri Nets and their corresponding Markov chains and present a failure analysis algorithm incorporating some critical factors. This algorithm can be implemented in diverse industrial applications.
\end{abstract}

\section{Introduction}

Failure can be defined as any unwanted deviation from the desired predetermined plan, which may lead to any kind of human injuries or damages of machines. In order to perform corrective actions, prioritizing failures is necessary. One of the most popular risk analysis procedures aiming to prioritizing failure states is Failure Mode and Effect Analysis (FMEA) which is based on three factors of severity, detectability, and occurrence of failure. This method was introduced in 1960s. Its wide applications are documented by Braglia et al. [1] and Stamatis [2]. Process of calculating risk priority numbers in this method consists of multiplication of the three-mentioned risk factors. It is apparent that various combinations of risk factors may lead to a constant risk priority number. This is the most critical challenge FMEA faces. However, there are many other weaknesses in this method which makes it impractical in real world problems $[3,4]$.

Up to now, many different approaches have been presented to overcome the limitations of FMEA. These methods rely on diverse techniques such as grey theory [4], Bayesian Nets [5], Monte Carlo simulation [6], Markov models [7], and fuzzy logic [8]. Among all these techniques, fuzzy logic has been extensively applied in risk analysis. Fuzzy logic is capable of handling vagueness of human suggestions; so that it is useful in prioritizing risky behaviors of the systems.

Fuzzy risk analysis is a new field firstly introduced by Schmucker [9]. This method is similar to traditional FMEA; however, risk parameters are fuzzy numbers. Fuzzy arithmetic is used to obtain fuzzy risk priority numbers. This method is rather versatile although suffers from ignoring many critical risk parameters. Using fuzzy risk analysis and fuzzy arithmetic, many different researches have been performed so far. Among them, combination of fuzzy risk analysis and ranking methods are extensively studied. Based on Schmucker [9], a fuzzy risk analysis method using signal/noise ratio was presented by Chen and Wang [10], in which fuzzy risk priority numbers were ranked by this ratio. Different kinds of fuzzy numbers such as generalized ones and their application in safety analysis are investigated by $\mathrm{S}$. M. Chen and J. H. Chen $[11,12]$. Application of similarity measures between fuzzy numbers is investigated in [11-13]. The fuzzy risk analysis method proposed by Schmucker [9] is widely utilized in so many researches performed so far. Fuzzy risk analysis methods are based on either ranking techniques or similarity measures, with differences only in prioritization style. In ranking based methods fuzzy risk priority numbers 
(FRPN) are ranked by different ranking techniques; while in similarity based techniques, the same FRPN are ranked by measuring the similarity level between FRPN and some predetermined linguistic variables.

Failure analysis techniques traditionally rely on static evaluation of systems, which fails in tracking risky behaviors. Hence, implementing dynamic tools to enable auditors to monitor systems dynamically seems beneficial. One of the most common tools in modeling dynamic systems are Petri Nets (PN) [21, 22]. PN are powerful modeling methods which can simulate behaviors of systems dynamically in such a way that different states of the system can be visualized. There are many different versions of Petri Nets including timed, stochastic, and colored. In stochastic Petri Nets firing of transitions depend on a stochastic variable with exponential distribution.

In this paper, we introduce a novel failure analysis method in which the Petri Net model of the system is constructed. This model includes different potential risks. According to isomorphism between live bounded stochastic Petri Nets and Markov chains, fuzzy steady state probabilities are calculated in the form of generalized trapezoidal fuzzy numbers. In these fuzzy probabilities, the weight of each fuzzy number is a combination of several new risk factors. Finally, these fuzzy probabilities are ranked by a new powerful fuzzy ranking method.

The rest of the paper is structured as follows. In Section 2, the bases of Stochastic Petri Nets (SPN) are given. SPN with fuzzy parameters and their application in safety analysis of the systems are summarized in Section 3. In Section 4, a novel ranking method of generalized fuzzy numbers is presented and compared with other methods in the field. In Section 5, we deal with a risk prioritizing method using SPN and generalized fuzzy numbers which opens a new field in risk prioritizing techniques literature. In Section 6, an illustrative example is presented to clarify the methods proposed in the paper. The paper is concluded in Section 7.

\section{Stochastic Petri Nets}

SPN are a family member of Petri Nets in which firing rates are exponentially distributed. Before introducing SPN, we present the definition of Petri Nets introduced by Petri [23]. Petri Net $(\mathrm{PN}) Z=(P, T, I, O, m)$ is a five-tuple, where

(1) $P=\left\{p_{1}, p_{2}, \ldots, p_{n}\right\}, n>0$ is a finite set of places pictured by circles,

(2) $T=\left\{t_{1}, t_{2}, \ldots, t_{s}\right\}, s>0$ is a finite set of transitions pictured by bars,

(3) $I=P \times T \rightarrow N$ is an input function that defines the set of directed $\operatorname{arcs}$ from $P$ to $T$ where $N=$ $\{0,1,2, \ldots\}$,

(4) $O=T \times P \rightarrow N$ is an output function that defines the set of directed arcs from $T$ to $P$,

(5) $m: P \rightarrow N$ is a marking whose $i$ th component represents the number of tokens in the ith place. An initial marking is denoted by $m_{0}$. The tokens are pictured by dots.
PN are able to model systems dynamically. One of the crucial drawbacks in utilizing ordinary $\mathrm{PN}$ is their inability in handling some important factors such as time and vagueness. To solve such problems various kinds of nets such as timed, fuzzy, and stochastic PN have been developed.

In SPN, the set of firing rates $\Lambda=\left(\lambda_{1}, \lambda_{2}, \ldots, \lambda_{s}\right)$ are exponentially distributed, such that each transition can be fired only after an exponentially distributed time delay with parameter $1 / \lambda$ elapses.

An important aspect of stochastic $\mathrm{PN}$ is their isomorphism with Markov chains. It has been proved that live and bounded SPN are isomorphic to continuous-time Markov chains [24]. This important property makes SPN analysis straightforward. In SPN, each marking of the net is equivalent to the states of its corresponding Markov chain. Therefore, some important factors, like steady state probabilities, are easily computed. Here, we present the formal definition of SPN.

A SPN $Z=\left(P, T, I, O, m_{0}, \Lambda\right)$ is a six-tuple, where

(1) $P=\left\{p_{1}, p_{2}, \ldots, p_{n}\right\}, n>0$ is a finite set of places,

(2) $T=\left\{t_{1}, t_{2}, \ldots, t_{s}\right\}, s>0$ is a finite set of transitions with $P \cup T \neq \varnothing$, and $P \cap T=\varnothing$,

(3) $I=P \times T \rightarrow N$ is an input function that defines the set of directed arcs from $P$ to $T$ where $N=$ $\{0,1,2, \ldots\}$,

(4) $O=T \times P \rightarrow N$ is an output function that defines the set of directed arcs from $T$ to $P$,

(5) $m: P \rightarrow N$ is a marking whose $i$ th component represents the number of tokens in the $i$ th place. An initial marking is denoted by $m_{0}$, and

(6) $\Lambda: T \rightarrow R^{+}$is a firing function whose $i$ th component represents the firing rate of the $i$ th transition where $\lambda_{i}$ denotes the firing rate of $t_{i}$ and $R^{+}$is the set of all possible real values.

Firing rules in SPN are simply similar to ordinary PN with a difference only in firing times of transitions. In SPN, when a transition is enabled, all tokens in the upstream places remain in their places until the firing time of the corresponding transition elapses; then the tokens deposited in upward places are removed and added to all downward places of that transition.

In order to construct the corresponding Markov chain of SPN, the reachability graph of the net must be constructed first. Then using (1), the steady state probabilities can be calculated,

$$
\Pi Q=0, \quad \sum_{i=0}^{s} \pi_{i}=1
$$

in which $\pi_{i}$ is the probability of being in state $M_{i}$; and $\Pi=\left(\pi_{1}, \pi_{2}, \ldots, \pi_{s}\right)$. From steady state distribution $\Pi$, this is possible to predict the performance of the system. For more details the reader is referred to Bause and Kritzinger [25]. 


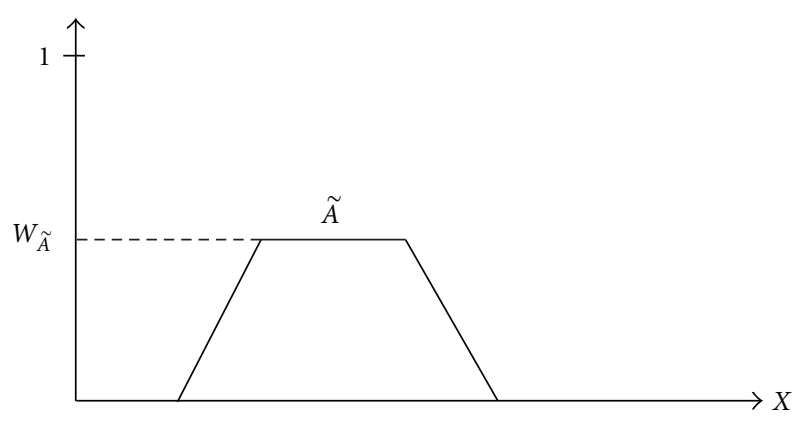

FIGURE 1: A generalized trapezoidal fuzzy number.

\section{Stochastic Petri Nets with Fuzzy Parameters}

Safety is the knowledge of utilizing recorded data to predict potential failure in future. In normal conditions, failures occur rarely. Thus, expertise in predicting the frequency of different failure states is necessary. Suggestions by experts are usually vague and do not include specific numerical values and are given often in linguistic variables. Classical mathematics faces difficulties in handling nondeterministic values. Fuzzy set theory is a powerful method for handling vague conditions.

In this paper, according to vagueness of experts' knowledge in predicting failure rates of different risky states, we propose a new approach to determine fuzzy steady state probabilities based on generalized fuzzy numbers.

As explained earlier, SPN are isomorphic to continuoustime Markov chains; thus steady state probabilities can be computed by dominating rules on Markov chains. We consider the failure rates of each potential risk in the system as a fuzzy number. Let us introduce some notations used in the rest of the paper; for more information, see [26].

Definition 1. A fuzzy number is a convex normalized fuzzy set $\widetilde{M}$ of the real line $\mathbb{R}$ such that

(1) there exists exactly one $x_{0} \in \mathbb{R}$ with $\mu_{\tilde{m}}\left(x_{0}\right)=1\left(x_{0}\right.$ is called the mean value of $\widetilde{M}$ );

(2) $\mu_{\tilde{m}}\left(x_{0}\right)$ is piecewise continuous.

For the sake of computational efficiency, some special forms of fuzzy numbers with triangular or trapezoidal membership functions are used. Sometimes, a more general form of fuzzy numbers is needed. For instance, in this paper, generalized fuzzy numbers are used with the extra feature of weighted membership functions. Their generality is according to the weight of the mean value or the supremum of their membership function. In Figure 1 a generalized trapezoidal fuzzy number is depicted.

Definition 2. An alpha cut ( $\alpha$-cut) of a fuzzy number $\tilde{A}$, if it is a subset of the set $\Omega$, is defined as:

$$
\tilde{A}(\alpha)=\{x \in \Omega \mid \tilde{A}(\alpha) \geq \alpha\} \quad 0<\alpha \leq 1 .
$$

On the other hand, each fuzzy number can be represented by its alpha cut. For example, in a fuzzy number $\widetilde{Q}$ we have

$$
\widetilde{Q}(\alpha)=\left[q_{1}(\alpha), q_{2}(\alpha)\right]
$$

in which values of $q_{1}(\alpha)$ and $q_{2}(\alpha)$ are the lower and upper bounds of this alpha cut, respectively.

Fuzzy arithmetic offers two concepts: extension principle and operations between alpha cuts. In this paper, we rely on the second concept since it can be adapted by the extension principle; in addition, incorporating alpha cuts is easier.

Let us consider two fuzzy numbers $\widetilde{A}$ and $\widetilde{B}$ and their alpha cuts $\tilde{A}(\alpha)=\left[a_{1}(\alpha), a_{2}(\alpha)\right]$ and $\widetilde{B}(\alpha)=\left[b_{1}(\alpha), b_{2}(\alpha)\right]$, respectively. Operations between fuzzy numbers in the framework of alpha cuts are

$$
\begin{aligned}
& \tilde{A}(\alpha)+\widetilde{B}(\alpha)=\left[a_{1}(\alpha)+b_{1}(\alpha), a_{2}(\alpha)+b_{2}(\alpha)\right], \\
& \tilde{A}(\alpha)-\widetilde{B}(\alpha)=\left[a_{1}(\alpha)-b_{2}(\alpha), a_{2}(\alpha)-b_{1}(\alpha)\right], \\
& \widetilde{A}(\alpha) \cdot \widetilde{B}(\alpha)=[c(\alpha), d(\alpha)]
\end{aligned}
$$

in which,

$$
\begin{aligned}
& c(\alpha)=\min \left\{a_{1}(\alpha) b_{1}(\alpha), a_{1}(\alpha) b_{2}(\alpha), a_{2}(\alpha) b_{1}(\alpha), a_{2}(\alpha) b_{2}(\alpha)\right\} \\
& d(\alpha)=\max \left\{a_{1}(\alpha) b_{1}(\alpha), a_{1}(\alpha) b_{2}(\alpha), a_{2}(\alpha) b_{1}(\alpha), a_{2}(\alpha) b_{2}(\alpha)\right\}
\end{aligned}
$$

The dividing operation among fuzzy numbers is defined as

$$
\frac{\widetilde{A}(\alpha)}{\widetilde{B}(\alpha)}=\left[a_{1}(\alpha), a_{2}(\alpha)\right] \cdot\left[\frac{1}{b_{2}(\alpha)}, \frac{1}{b_{1}(\alpha)}\right] .
$$

\section{Ranking Method of Generalized Fuzzy Numbers}

Application of fuzzy sets theory in reliability and safety engineering has been an active field of research in recent years. One of the most alluring fuzzy techniques is ranking of fuzzy numbers. Chen and Wang [10] proposed a fuzzy risk analysis method based on signal/noise ratio. For more details, reader is referred to [11-13].

In this section, we propose a novel approach for ranking generalized trapezoidal fuzzy numbers. This approach will be used for risk analysis in the following sections. The algorithm is as follows.

Step 1. Consider the generalized fuzzy number $\widetilde{A}_{i}=$ $\left(a_{i 1}, a_{i 2}, a_{i 3}, a_{i 4} ; w_{\tilde{A}}\right)$. Use (7) for standardizing the generalized fuzzy number

$$
\begin{aligned}
\tilde{A}_{i^{*}} & =\left(\frac{a_{i 1}}{k}, \frac{a_{i 2}}{k}, \frac{a_{i 3}}{k}, \frac{a_{i 4}}{k} ; w_{\tilde{A}_{i^{*}}}\right), \\
k & =\max _{i j}\left(\left[\left|a_{i j}\right|\right\rceil, 1\right) .
\end{aligned}
$$


TABLe 1: Comparison results of Figure 2.

\begin{tabular}{lcc}
\hline Ranking method & $\widetilde{A}$ & $\widetilde{B}$ \\
\hline S. M. Chen and J. H. Chen [11] & $\mathbf{0 . 1 3 7 5}$ & $\mathbf{0 . 1 3 7 5}$ \\
The proposed method & 1.68486 & 0.30964 \\
\hline
\end{tabular}

Step 2. Calculate the center of gravity point of the standard fuzzy number, (7),

$$
\begin{gathered}
y_{\widetilde{A}_{i^{*}}}= \begin{cases}\frac{\left(\left(a_{i 3}-a_{i 2}\right) /\left(a_{i 4}-a_{i 1}\right)+2\right) w_{\widetilde{A}_{i^{*}}}}{6} & a_{i 1} \neq a_{i 4}, \\
\frac{w_{\widetilde{A}_{i^{*}}}}{2}, & a_{i 1}=a_{i 4},\end{cases} \\
x_{\widetilde{A}_{i^{*}}}=\frac{y_{\widetilde{A}_{i^{*}}}\left(a_{i 3}+a_{i 2}\right)+\left(a_{i 4}+a_{i 1}\right)\left(w_{\widetilde{A}_{i^{*}}}-y_{\widetilde{A}_{i^{*}}}\right)}{2 w_{\widetilde{A}_{i^{*}}}} .
\end{gathered}
$$

Step 3. Calculate the standard deviation of each standardized fuzzy number,

$$
\operatorname{STD}_{\widetilde{A}_{i^{*}}}=\sqrt{\frac{\sum_{j=1}^{4}\left(a_{i j}^{*}-x_{\widetilde{A}_{*}}\right)^{2}}{4-1}} .
$$

It is apparent that the interval of the obtained standard deviation is $[0,1.1547]$. Variation of a crisp value is zero and variation of the generalized fuzzy number $(-1,-1,-1$, $-1: w)$ is 1.1547 .

Step 4. Calculate the difference of $x$ coordinate of each fuzzy number from the least value,

$$
\alpha=\frac{x^{*}-x_{\min }^{*}}{x_{\max }^{*}-x_{\min }^{*}} \quad \text { if } x_{\min }^{*} \neq x_{\max }^{*}
$$

in which

$$
\begin{aligned}
x_{\max }^{*} & =\operatorname{Max}\left\{x_{\widetilde{A}_{1^{*}}}, x_{\widetilde{A}_{2^{*}}}, \ldots, x_{\widetilde{A}_{n^{*}}}\right\}, \\
x_{\min }^{*} & =\operatorname{Min}\left\{x_{\widetilde{A}_{1^{*}}}, x_{\widetilde{A}_{2^{*}}}, \ldots, x_{\widetilde{A}_{n^{*}}}\right\}, \\
\alpha & =0 \quad \text { if } x_{\min }^{*}=x_{\max }^{*} \neq 0, \\
\alpha & =0.5 \quad \text { if } x_{\min }^{*}=x_{\max }^{*}=0 .
\end{aligned}
$$

Step 5. Calculate the ranking value of each standard generalized trapezoidal fuzzy number,

$$
\operatorname{Rank}_{\widetilde{A}_{i^{*}}}=\frac{x_{\widetilde{A}_{i^{*}}}\left(w_{\widetilde{A}_{i^{*}}}+1\right)+\alpha}{1+\operatorname{STD}_{\widetilde{A}_{i^{*}}}} .
$$

Notation 1. The proposed ranking method concentrates on crisp value of the fuzzy number in contrast to its deviation.

Example 3. In order to demonstrate the capabilities of the method we have compared the ranking output with six other important and common ranking techniques. This comparison is performed for two groups of fuzzy numbers, Figures 2 and 3.

The ranking is shown and compared for the first group in Table 1. This is clear that S. M. Chen and J. H. Chen [11]

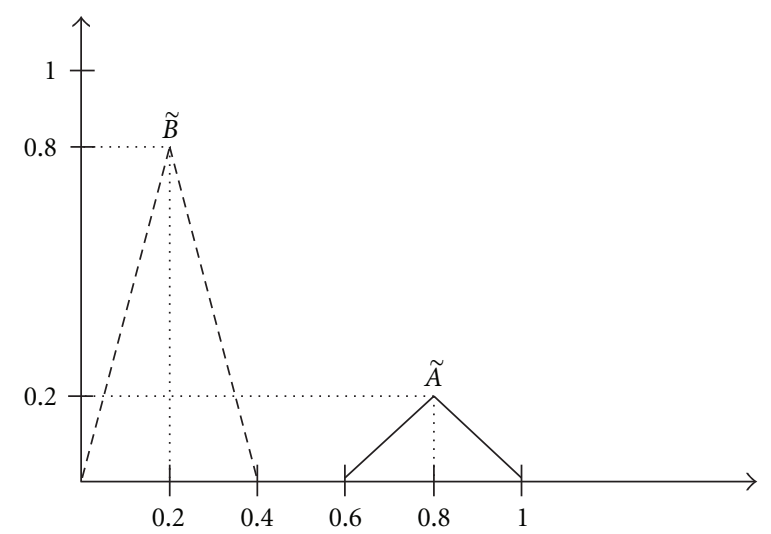

Figure 2: Group 1: Two generalized triangular fuzzy numbers. Both deviations and (mean value $\times$ weight) are the same for both numbers.

method fails to rank the fuzzy numbers $\widetilde{A}$ and $\widetilde{B}$. This is the case whenever the deviations and (mean value $\times$ weight) are the same for fuzzy numbers, [27].

It is noteworthy to mention that in our proposed method, crisp value of the fuzzy number has priority over its deviation and spread. Therefore, the number $\tilde{A}$ has priority over the other fuzzy numbers.

The second group, Figure 3, consists of eight sets of fuzzy numbers with different shapes and deviations suitable to evaluate our ranking algorithm [12]. Table 2 gives the result of ranking for this group, ranked using the proposed method and seven different algorithms presented in the literature. The highlighted items indicate invalid ranking by different algorithms. Cheng's method [14] and Chu's method [15] cannot rank fuzzy numbers of the second and third sets. Murakami's technique [16] gives the same result for the two members of set 3. Yager's method [17], as one of the most common ranking techniques, gives the same result for set 2 , set 3 , and set 4 . This shows that this algorithm is not capable of ranking fuzzy numbers in general. All these algorithms fail in ranking of fuzzy numbers in the set 5. S. J. Chen's and S. M. Chen's method [18] ranks the numbers correctly; however, the ranking scores are very close to each other. For sets 2 and 6, Lee and Chen' method [19] ranks the fuzzy numbers in an incorrect order.

As noted before, the main focus of the method proposed in the current paper is on crisp values of fuzzy numbers; that is why S. M. Chen's and J. H. Chen's method [11] has a valid but different ranking result. The latter method puts priority on variation and spread of numbers instead of their crisp values.

According to the results presented in Table 2, Figure 5 shows the percentage of correct answers of the compared methods. It can be seen that only the proposed method and the approach proposed by S. J. Chen and S. M. Chen [18] has successfully solved the entire fuzzy sets of Figure 3.

Example 4. One of the latest fuzzy risk analysis approaches, based on ranking fuzzy numbers, is presented in [20], in which the ranking technique is based on the areas between 


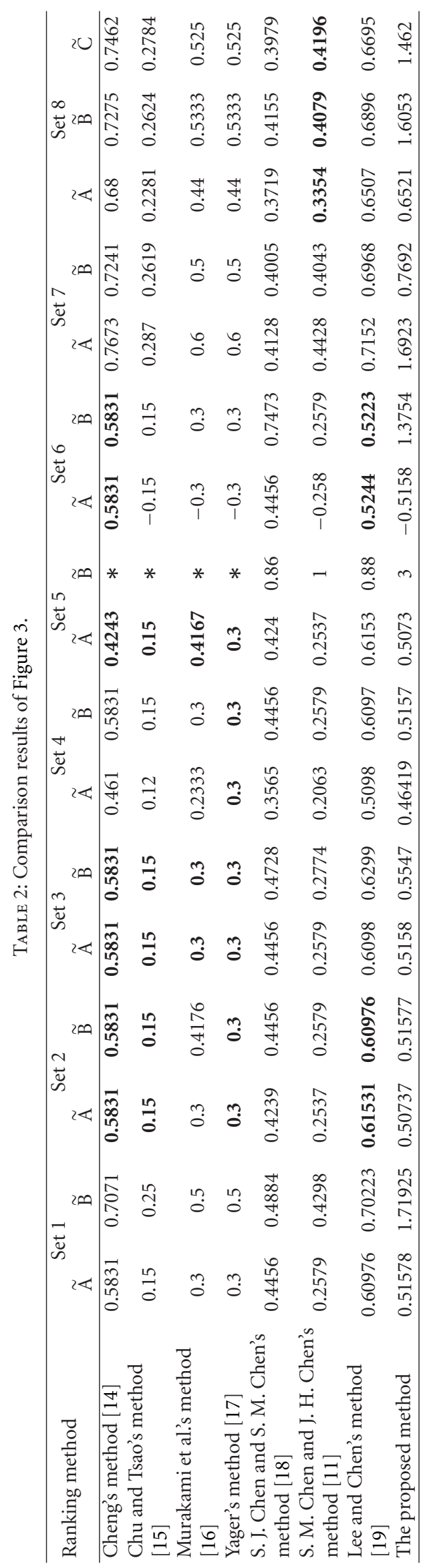



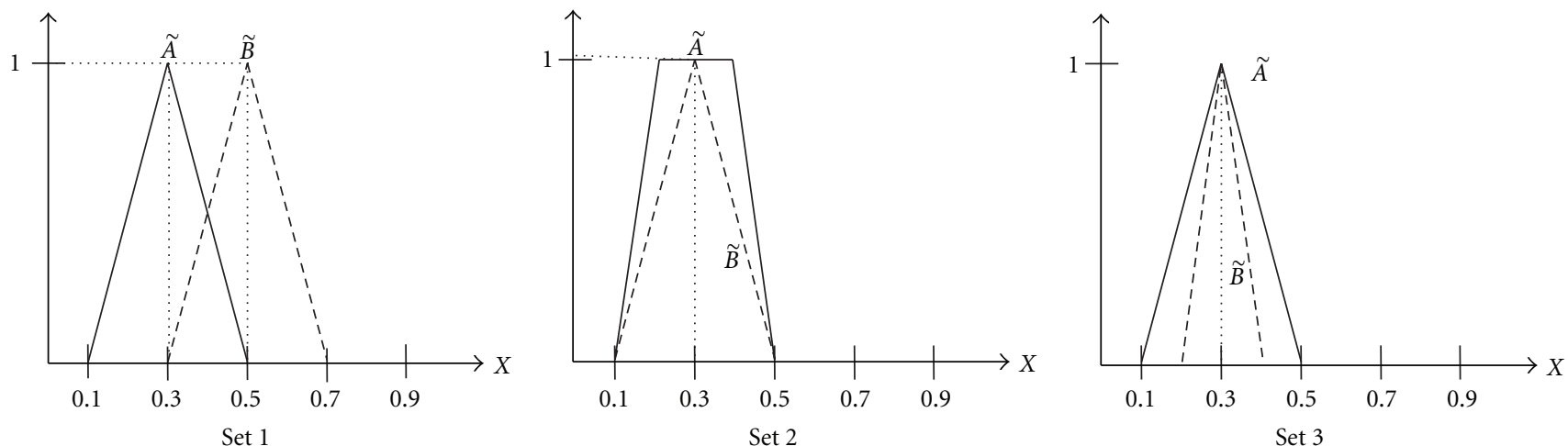

$\begin{aligned} \widetilde{A} & =(0.1,0.3,0.3,0.5 ; 1) \\ \widetilde{B} & =(0.3,0.5,0.5,0.7 ; 1)\end{aligned}$

$\widetilde{A}=(0.1,0.2,0.4,0.5 ; 1)$

$\widetilde{B}=(0.1,0.3,0.3,0.5 ; 1)$

$\widetilde{A}=(0.1,0.3,0.3,0.5 ; 1)$

$\widetilde{B}=(0.2,0.3,0.3,0.4 ; 1)$
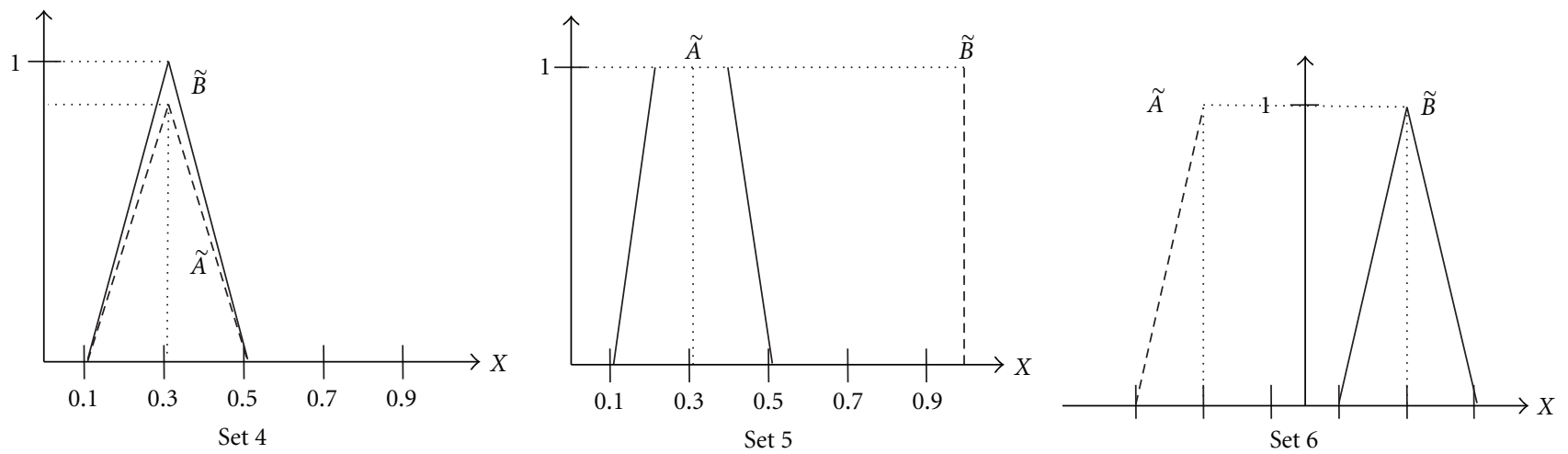

$\widetilde{A}=(0.1,0.3,0.3,0.5 ; 0.8)$
$\widetilde{B}=(0.1,0.3,0.3,0.5 ; 1)$

$\widetilde{A}=(0.1,0.2,0.4,0.5 ; 1)$

$\widetilde{A}=(-0.5,-0.3,-0.3,-0.1 ; 1)$

$\widetilde{B}=(1,1,1,1 ; 1)$

$\widetilde{B}=(0.1,0.3,0.3,0.5 ; 1)$
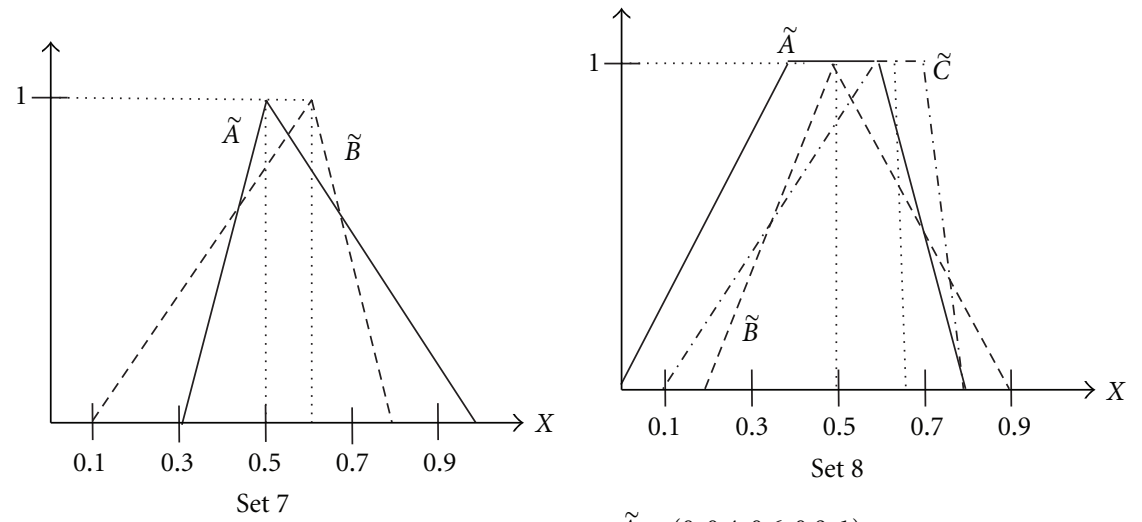

$\widetilde{A}=(0.3,0.5,0.5,1 ; 1)$

$\widetilde{A}=(0,0.4,0.6,0.8 ; 1)$

$\widetilde{B}=(0.2,0.5,0.5,0.9 ; 1)$

$\widetilde{B}=(0.1,0.6,0.6,0.8 ; 1)$

$\widetilde{C}=(0.1,0.6, .0 .7,0.8 ; 1)$

FIgURE 3: Group 2: seven sets of fuzzy numbers used to test the ranking algorithm [12].

the left and the right parts of the membership function of each fuzzy number with 1 and -1 as possible infimum and supremum values of fuzzy numbers. However this approach is not able to deal with symmetric fuzzy numbers. In such cases, the result of ranking for each symmetric fuzzy number will be zero. For example, consider two symmetric generalized fuzzy numbers $\widetilde{A}=(-0.8,0,0,0.8 ; 0.5)$ and $\widetilde{B}=$ $(-0.5,0,0,0.5 ; 0.7)$ as depicted in Figure 4 . Table 3 compares the results using Chen and Sanguansat method [20] and the current algorithm. It is clear that the Chen and Sanguansat method [20] is not able to rank symmetric generalized fuzzy numbers. 


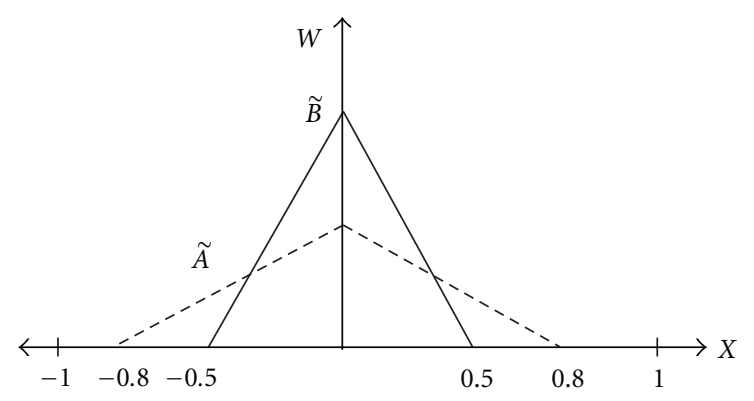

FIgURe 4: Two symmetric fuzzy numbers.

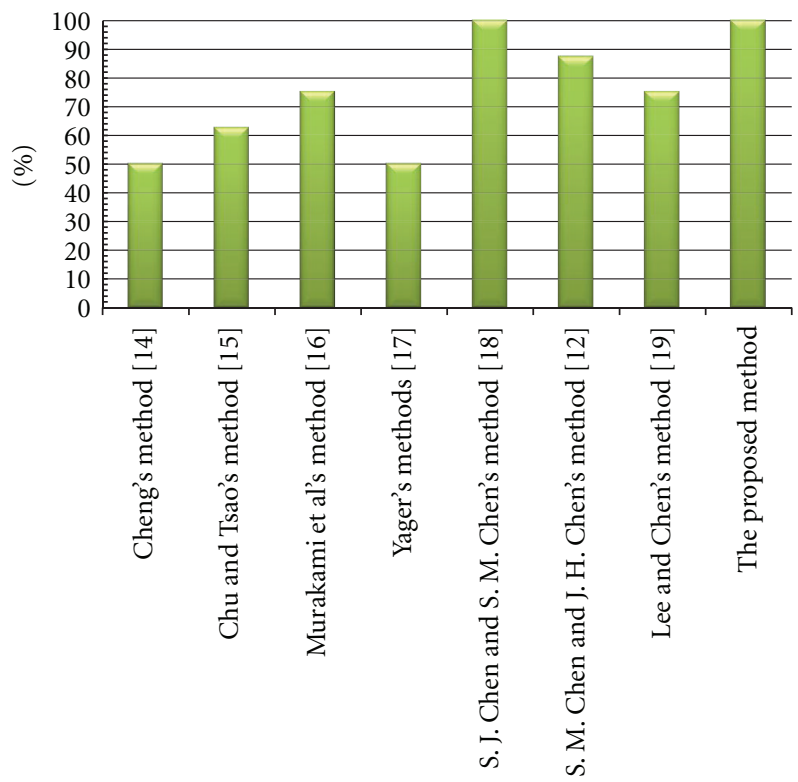

FIGURE 5: Percentage of correctness of the compared method according to Table 2 .

TABLE 3: Ranking of numbers given in Figure 4.

\begin{tabular}{lcc}
\hline Ranking method & $\widetilde{A}$ & $\widetilde{B}$ \\
\hline Chen and Sanguansat [20] & $\mathbf{0}$ & $\mathbf{0}$ \\
Current paper & 0.3024 & 0.3464 \\
\hline
\end{tabular}

\section{Risk Prioritizing}

Modeling complex systems using ordinary Petri Nets is a daunting task, since implementation of some concepts such as time, possibility, and probability is not considered in the initial definition of ordinary PN. Generally, analysis of complex systems includes two kinds of uncertainties [18]: stochastic situations and fuzzy states. For stochastic situations the behavior of system parameters is described by probability distribution functions. In other words, this kind of uncertainty models randomness. On the other hand, uncertainty in fuzzy form models the level of measurement accuracy using linguistic structures and insufficient information. There are many inaccuracy sources in systems, such as inaccurate internal operations. In some cases, uncertainty is the result of both randomness and inaccuracy, simultaneously. In stochastic PN, where time is the only existing stochastic parameter, system delays can be described by probability functions. It is worth to note that during system analysis, existing uncertainties may be hidden in the final results. Therefore, utilizing fuzzy set theory is an important alternative to overcome this drawback.

Although the dominant paradigm in describing uncertainties of models is stochastic modeling based upon probability, using such models is only appropriate for describing stochastic states among entire uncertain situations. This is more important when considering inaccuracy of some data which are not instinctively statistical [28].

Here, a comprehensive approach to prioritize different risk states of the system is presented. In this approach, by utilizing probability determination via steady state probabilities of stochastic PN, fuzzy probabilities of occurrence of each failure are calculated. In our approach, parameters of the exponential distribution are deemed to be generalized fuzzy numbers. Eventually, values of the resulting fuzzy probabilities are ranked by the novel ranking method presented in Section 3. In the following, we present the process of prioritization of failure modes. Our approach has three stages where each stage consists of several substages. This approach extends the method proposed in [29] to a risk analysis method.

Stage 1. (1) Modeling the desired system using Petri Nets and determining the entire failure modes and allocating exponential firing times to considered transitions.

(2) Constructing reachability graph of the net and determining all states.

(3) Incorporating (1) in order to calculate the steady state probabilities of the system parametrically based on exponential rates of system transitions.

Stage 2. (1) Conversion of parametric probabilities of (3) in Stage 1 to triangular fuzzy numbers considering each parameter.

(2) Determination of fuzzy probabilities based on alpha cuts of each fuzzy number using (4) to (6).

(3) Calculation of each probabilistic value $\left(\pi_{i}\right)$ and determination of maximum and minimum values of this probability $(\alpha=0)$. When $\alpha=0$, each probability $\pi_{i}$ must be in interval $[0,1]$ to be feasible; thus next steps are proceeded if this condition does not hold.

(4) Operations among fuzzy numbers using alpha cuts depend on minimum and maximum operators. This will provide a larger interval during the calculation. Theoretically, $\alpha=0$ cut of a fuzzy number gives the largest interval of the number. Since our aim is to find the fuzzy probability values, the largest possible value of fuzzy numbers must be constrained to $[0,1]$. Therefore, our aim is to find the shortest alpha:

$$
\begin{array}{ll}
\text { Min } & (Z)=\alpha \\
\text { St. } & \pi_{i}^{+}(\alpha) \leq 1 \\
& \pi_{i}^{-}(\alpha) \geq 0 \\
& \pi_{i}^{-}(\alpha) \leq \pi_{i}^{+}(\alpha) \\
& 0 \leq \alpha \leq 1 .
\end{array}
$$


TABLE 4: Classification of safety severity of each failure mode.

\begin{tabular}{lr}
\hline State & $S_{j 1}$ \\
\hline Very dangerous, without warning and periodical & 1 \\
inspection & 0.9 \\
Very dangerous, without periodical inspection & 0.8 \\
Very dangerous with automatic warning system & 0.7 \\
$\begin{array}{l}\text { Dangerous, without warning and periodical } \\
\text { inspection }\end{array}$ & \\
$\begin{array}{l}\text { Dangerous, with periodical inspection or warning } \\
\text { system }\end{array}$ & 0.6 \\
$\begin{array}{l}\text { Average danger, without warning system and } \\
\text { periodical inspection }\end{array}$ & 0.5 \\
Average danger with warning system and & \\
periodical inspection & 0.4 \\
Low danger, without warning system or & \\
periodical inspection & 0.3 \\
Low danger, with warning system or periodical & \\
inspection & 0.2 \\
Without any important risk & 0.1 \\
No safety risk & 0
\end{tabular}

TABle 5: Classification operational dependability between failure modes.

\begin{tabular}{lc}
\hline State & $S_{j 2}$ \\
\hline Very high, many numbers of machines malfunctioned & 1 \\
Very high, loss of quality in many of machines & 0.9 \\
High, an entire machine is off, a bottleneck is made for & 0.8 \\
$\begin{array}{l}\text { some sets of machines } \\
\text { High, an entire machine is off }\end{array}$ & 0.7 \\
$\begin{array}{l}\text { Average to high, the initial performance of machine is } \\
\text { lost but some tasks are possible to perform }\end{array}$ & 0.6 \\
$\begin{array}{l}\text { Average, the initial performance of machine is lost but } \\
\text { some tasks are possible to perform }\end{array}$ & 0.5 \\
$\begin{array}{l}\text { Average, machine loses its functionality in some specific } \\
\text { tasks }\end{array}$ & 0.4 \\
Low, quality decrease in secondary functions of & \\
machines & 0.3 \\
Low, machine loses a little part of its functionality & 0.2 \\
Very low & 0.1 \\
No dependability & 0 \\
\hline
\end{tabular}

Stage 3. (1) Converting each resulting failure probability to a generalized fuzzy number. This conversion is performed by combining severity index of each failure mode with fuzzy probabilities calculated in Stage 2. In this research, we have considered five critical factors to determine occurrence weight of each failure mode. These factors are maintenance costs, operational dependability, safety, failure detection methods, and repairing time.

In order to obtain these factors easily, we have provided some linguistic variables with their corresponding weights presented in Tables 4, 5, 6, 7, and 8. Finally, these critical factors must be combined to get the severity factor of each failure mode. This process includes a multiplication of these variables as

$$
S_{j}=S_{j 1} \times S_{j 2} \times S_{j 3} \times S_{j 4} \times S_{j 5}
$$

TABLE 6: Classification of detection and identification of failure modes.

\begin{tabular}{lc}
\hline State & $S_{j 3}$ \\
\hline Uncertainty & 1 \\
Very unlikely & 0.9 \\
unlikely & 0.8 \\
Very low & 0.7 \\
low & 0.6 \\
Average & 0.5 \\
Average to high & 0.4 \\
high & 0.3 \\
Very high & 0.2 \\
Nearly definite & 0.1 \\
Completely definite & 0 \\
\hline
\end{tabular}

TABLE 7: Classification of repairing costs of failure modes.

\begin{tabular}{lc}
\hline State & $S_{j 4}$ \\
\hline Is not worth to fix & 1 \\
Hardly worth to fix & 0.9 \\
Extreme & 0.8 \\
High & 0.7 \\
Average high & 0.6 \\
Average & 0.5 \\
low & 0.4 \\
Average low & 0.3 \\
Low & 0.2 \\
Low importance & 0.1 \\
No cost & 0 \\
\hline
\end{tabular}

TABLE 8: Classification of repairing time of a failure mode.

\begin{tabular}{lc}
\hline State & $S_{j 5}$ \\
\hline Very time consuming, no worth to fix & 1 \\
Long fixing time, hardly worth to fix & 0.9 \\
Very time consuming to fix & 0.8 \\
Long fixing time & 0.7 \\
Average to high fixing time & 0.6 \\
Average fixing time & 0.5 \\
Short fixing time & 0.4 \\
Fairly short fixing time & 0.3 \\
Very short fixing time & 0.2 \\
Fixing time not very important & 0.1 \\
Fixing time negligible & 0 \\
\hline
\end{tabular}

in which $S_{i j}$ represents the value of $i$ th factor from five critical factors influencing failure $j$ and $S_{j}$ represents the final severity measure of the failure $j$.

(2) Ranking each generalized fuzzy probability provided in (1) in Stage 3 using the ranking technique proposed in Section 3. 
Table 9: Description of Places of Figure 7.

\begin{tabular}{ll}
\hline Place & Description \\
\hline$P_{1}$ & Work-piece ready \\
$P_{2}$ & Robot in progress \\
$P_{3}$ & Machine in process \\
$P_{4}$ & Robot in repair \\
$P_{5}$ & Machine in repair \\
$P_{6}$ & Robot idle \\
$P_{7}$ & Machine idle \\
\hline
\end{tabular}

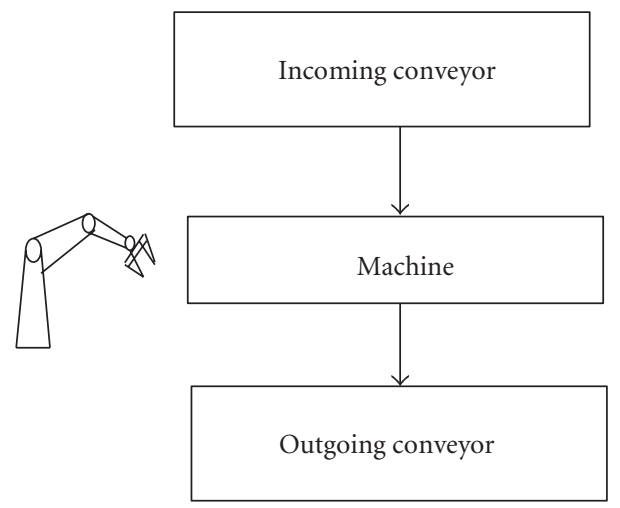

Figure 6: A flexible manufacturing cell.

\section{An Illustrative Example}

In this section the approaches proposed in the paper are applied to a flexible manufacturing (FM) cell, adopted from [30]. This cell is of course adapted with the nature of failure analysis.The cell, Figure 6, has one incoming and one outgoing conveyor, one robotic arm, and one processing machine. Work-pieces enter the cell by an incoming conveyor and the robot (R) loads them to the machine (M). Since we want to consider different potential failure modes of the system, a breakdown loop is considered for the robot and processing machine which consists of a breakdown and a repair transition with their corresponding rates. When processing on $\mathrm{M}$ is over, it is unloaded by $\mathrm{R}$ and the workpiece will go to the outgoing conveyor. Now suppose that

(1) the processing machine can have failure modes. M takes two time units to breakdown and a quarter time unit to be repaired. Therefore, the average failure and repair rates are 0.5 and 4 , respectively;

(2) robot loading and taking work-piece rate is 45 per unit time. Also its unloading rate plus average rate of $\mathrm{M}$ processing is 8 per unit time. Robot is not failure free; hence, breakdown and repair rates, for the robot, are 0.4 and 5 , respectively;

(3) time delays considered in this example are entirely exponential.

Now, the problem is to find fuzzy risk probabilities based on fuzzy steady state probabilities and risk parameters introduced earlier; then prioritizing them on the basis of

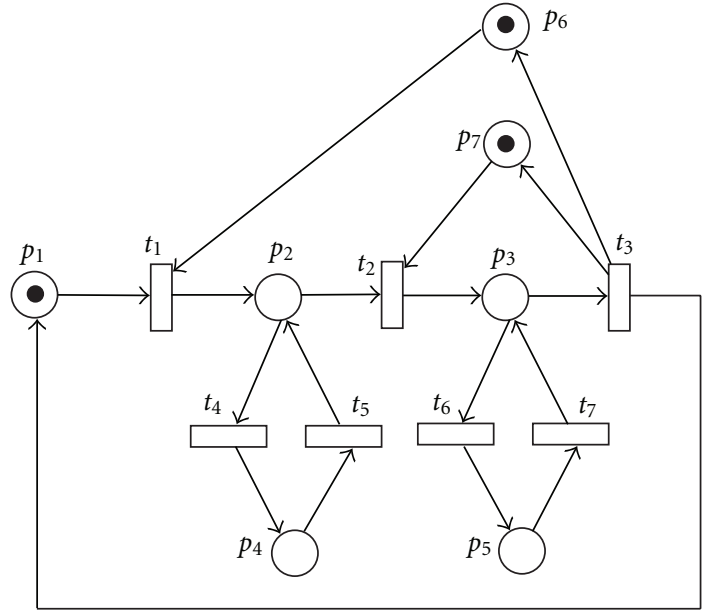

Figure 7: The Petri Net model of a flexible manufacturing cell.

the presented ranking technique. The Petri Net model of the system is live and bounded. Therefore, it is isomorphic with its corresponding Markov chain and the analysis can be performed by analysis of the Markov chain.

In order to find fuzzy probabilities, we have to delineate the reachability and the Markov chain of the corresponding stochastic Petri Net of the FM cell.

The description of places and transitions is represented in Tables 9 and 10, respectively. Transitions firing rates are displayed in Table 11.

In order to apply the proposed method, transition firing rate must be converted to fuzzy form and their corresponding alpha cuts must be obtained. The results of this transformation are shown in Table 12.

Based on Figure 8, parametric steady state probabilities are calculated using (1),

$$
\begin{aligned}
& \left(\pi_{0}, \pi_{1}, \pi_{2}, \pi_{3}, \pi_{4}\right) \\
& \quad \times\left[\begin{array}{ccccc}
-\lambda_{1} & \lambda_{1} & 0 & 0 & 0 \\
0 & -\lambda_{2}-\lambda_{4} & \lambda_{4} & \lambda_{2} & 0 \\
0 & \lambda_{5} & -\lambda_{5} & 0 & 0 \\
\lambda_{3} & 0 & 0 & -\lambda_{3}-\lambda_{6} & \lambda_{6} \\
0 & 0 & 0 & \lambda_{7} & -\lambda_{7}
\end{array}\right]=0
\end{aligned}
$$

$\pi_{0}+\pi_{1}+\pi_{2}+\pi_{3}+\pi_{4}=1$.

The resulting parametric steady state probabilities are

$$
\Pi=\left[\begin{array}{l}
\pi_{0}=\frac{\lambda_{2} \lambda_{3} \lambda_{5} \lambda_{7}}{\lambda} \\
\pi_{1}=\frac{\lambda_{1} \lambda_{3} \lambda_{5} \lambda_{7}}{\lambda} \\
\pi_{2}=\frac{\lambda_{1} \lambda_{3} \lambda_{4} \lambda_{7}}{\lambda} \\
\pi_{3}=\frac{\lambda_{1} \lambda_{2} \lambda_{5} \lambda_{7}}{\lambda} \\
\pi_{4}=\frac{\lambda_{1} \lambda_{2} \lambda_{5} \lambda_{6}}{\lambda}
\end{array}\right]
$$




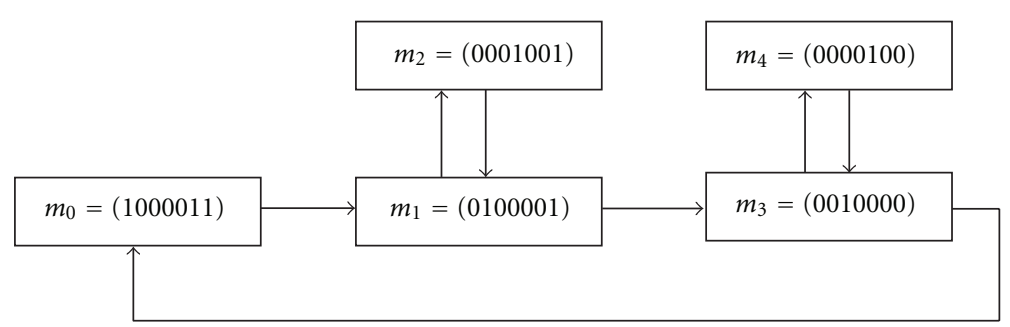

Figure 8: Markov chain and reachability graph of the modeled system.

where $\lambda=\lambda_{2} \lambda_{3} \lambda_{5} \lambda_{7}+\lambda_{1} \lambda_{3} \lambda_{5} \lambda_{7}+\lambda_{1} \lambda_{3} \lambda_{4} \lambda_{7}+\lambda_{1} \lambda_{2} \lambda_{5} \lambda_{7}+$ $\lambda_{1} \lambda_{2} \lambda_{5} \lambda_{6}$. After obtaining parametric steady state probabilities, they have to be converted to their corresponding fuzzy form. By applying fuzzified transition firing rates in steady state probabilities, fuzzy steady state probabilities are calculated. Therefore, the alpha cut representations of fuzzy steady state probabilities are

$$
\pi_{0}=\left[\frac{10 \alpha^{4}+175 \alpha^{3}+1100 \alpha^{2}+2975 \alpha+2940}{131 \alpha^{4}-2964.5 \alpha^{3}+25107 \alpha^{2}-94312.5 \alpha+132577.5}\right.
$$$$
\left.\frac{10 \alpha^{4}-255 \alpha^{3}+2390 \alpha^{2}-9795 \alpha+14850}{131 \alpha^{4}+1916.5 \alpha^{3}+9464 \alpha^{2}+25268.9 \alpha+22760.5}\right],
$$$$
\pi_{1}=\left[\frac{10 \alpha^{4}+175 \alpha^{3}+1100 \alpha^{2}+2975 \alpha+2940}{131 \alpha^{4}-2964.5 \alpha^{3}+25107 \alpha^{2}-94312.5 \alpha+132577.5} ;\right.
$$$$
\left.\frac{10 \alpha^{4}-255 \alpha^{3}+2390 \alpha^{2}-9795 \alpha+14850}{131 \alpha^{4}+1916.5 \alpha^{3}+9464 \alpha^{2}+25268.9 \alpha+22760.5}\right],
$$$$
\pi_{2}=\left[\frac{\alpha^{4}+16.5 \alpha^{3}+96.5 \alpha^{2}+241.5 \alpha+220.5}{131 \alpha^{4}-2964.5 \alpha^{3}+25107 \alpha^{2}-94312.5 \alpha+132577.5} ;\right.
$$$$
\left.\frac{\alpha^{4}+24.5 \alpha^{3}+219.5 \alpha^{2}-857.5 \alpha+1237.4}{131 \alpha^{4}+1916.5 \alpha^{3}+9464 \alpha^{2}+25268.9 \alpha+22760.5}\right],
$$$$
\pi_{3}=\left[\frac{100 \alpha^{4}+1400 \alpha^{3}+7325 \alpha^{2}+16975 \alpha+14700}{131 \alpha^{4}-2964.5 \alpha^{3}+25107 \alpha^{2}-94312.5 \alpha+132577.5} ;\right.
$$$$
\left.\frac{100 \alpha^{4}-2200 \alpha^{3}+18125 \alpha^{2}-66275 \alpha+90750}{131 \alpha^{4}+1916.5 \alpha^{3}+9464 \alpha^{2}+25268.9 \alpha+22760.5}\right],
$$$$
\pi_{4}=\left[\frac{10 \alpha^{4}+150 \alpha^{3}+842.5 \alpha^{2}+2102.4 \alpha+1960}{131 \alpha^{4}-2964.5 \alpha^{3}+25107 \alpha^{2}-94312.5 \alpha+132577.5} ;\right.
$$$$
\left.\frac{10 \alpha^{4}-230 \alpha^{3}+1982.5 \alpha^{2}-7590 \alpha+10890}{131 \alpha^{4}+1916.5 \alpha^{3}+9464 \alpha^{2}+25268.9 \alpha+22760.5}\right] .
$$

Since the value of fuzzy probabilities must be in interval $[0,1]$, we have to observe if they are out of this interval. When $\alpha=0$ all the minimum and maximum bounds of each fuzzy number must be in $[0,1]$; however it is apparent that $\pi_{3}^{+}(\alpha)$ exceeds 1 . On the other hand, all $\pi_{i}^{-}(\alpha)$ are positive.
TABLE 10: Description of transitions in Figure 7.

\begin{tabular}{ll}
\hline Transition & Description \\
\hline$T_{1}$ & Robot taking part \\
$T_{2}$ & Machine is processing \\
$T_{3}$ & Processing is finished and robot is unloading \\
$T_{4}$ & Robot breakdown \\
$T_{5}$ & Robot in repair \\
$T_{6}$ & Machine breakdown \\
$T_{7}$ & Machine in repair \\
\hline
\end{tabular}

Therefore, the LP model of the problem, using (13), is given as:

$$
\begin{aligned}
& \text { Min } Z=\alpha \\
& 121 \alpha^{4}+2171.5 \alpha^{3}+7074 \alpha^{2}+35063.9 \alpha+7910.5 \geq 0 \\
& 130 \alpha^{4}+1941 \alpha^{3}+9244.5 \alpha^{2}+26126.4 \alpha+21523 \geq 0 \\
& 31 \alpha^{4}+4116.5 \alpha^{3}-8661 \alpha^{2}+91543.9 \alpha-67990 \geq 0 \\
& 121 \alpha^{4}+2146.5 \alpha^{3}+7481.5 \alpha^{2}+32858.9 \alpha+11870.5 \geq 0 \\
& 0 \leq \alpha \leq 1 .
\end{aligned}
$$

Using LP software or spreadsheets like Excel, we can find the optimal value of $\alpha$ as 0.779 . Thus the feasible fuzzy steady state probabilities will be as given in, Table 13 .

In the next stage, the obtained fuzzy probabilities must be converted to generalized fuzzy numbers. According to Figure 8, we have two risky states in our model, $m_{2}$ and $m_{4}$, so we just deal with them. We call $m_{2}$ and $m_{4}$ as risky states $s_{1}$ and $s_{2}$, respectively. Based on our intuition, we consider risk factors for both $s_{1}$ and $s_{2}$, as in Table 14 .

The weights of both risky states are

$$
\begin{aligned}
& S_{1}=0.6 \times 0.8 \times 0.2 \times 0.8 \times 0.7=0.05376, \\
& S_{2}=0.8 \times 0.8 \times 0.2 \times 0.7 \times 0.7=0.06272 .
\end{aligned}
$$

Finally, the resulting generalized fuzzy probability numbers are (Figures 9-10):

$$
\begin{gathered}
\pi_{2}=(0.0065,0.0097,0.0097,0.0144 ; 0.05376), \\
\pi_{4}=(0.0573,0.085,0.085,0.0124 ; 0.06272) .
\end{gathered}
$$


TABLE 11: Transition firing rates of Figure 7.

\begin{tabular}{llc}
\hline Transition & Description & Rate \\
\hline$T_{1}$ & Taking part by R & 45 \\
$T_{2}$ & R loading & 45 \\
$T_{3}$ & M process finished and R is unloading & 8 \\
$T_{4}$ & R breakdown & 0.4 \\
$T_{5}$ & R repaired & 5 \\
$T_{6}$ & M breakdown & 0.5 \\
$T_{7}$ & M repaired & 4 \\
\hline
\end{tabular}

TABle 12: Fuzzified transitions firing rates with their alpha cuts.

\begin{tabular}{lc}
\hline Fuzzified rates & Alpha-cut representation \\
\hline$\lambda_{1}=(35,45,55)$ & $\lambda_{1}=(35+10 \alpha ; 55-10 \alpha)$ \\
$\lambda_{2}=(35,45,55)$ & $\lambda_{2}=(35+10 \alpha ; 55-10 \alpha)$ \\
$\lambda_{3}=(7,8,9)$ & $\lambda_{3}=(7+\alpha ; 9-\alpha)$ \\
$\lambda_{4}=(0.3,0.4,0.5)$ & $\lambda_{4}=(0.3+0.1 \alpha ; 0.5-0.1 \alpha)$ \\
$\lambda_{5}=(4,5,6)$ & $\lambda_{5}=(4+\alpha ; 6-\alpha)$ \\
$\lambda_{6}=(0.4,0.5,0.6)$ & $\lambda_{6}=(0.4+0.1 \alpha ; 0.6-0.1 \alpha)$ \\
$\lambda_{7}=(3,4,5)$ & $\lambda_{6}=(3+\alpha ; 5-\alpha)$ \\
\hline
\end{tabular}

TABLE 13: Fuzzy steady state probabilities.

\begin{tabular}{lc}
\hline$\pi_{i}$ & Fuzzy number \\
\hline$\pi_{0}$ & {$[0.082,0.121,0.174]$} \\
$\pi_{1}$ & {$[0.082,0.121,0.174]$} \\
$\pi_{2}$ & {$[0.0065,0.0097,0.0141]$} \\
$\pi_{3}$ & {$[0.453,0.68,1]$} \\
$\pi_{4}$ & {$[0.0573,0.085,0.124]$} \\
\hline
\end{tabular}

TABLE 14: The considered risk factors for $s_{1}$ and $s_{2}$.

\begin{tabular}{lc}
\hline$S_{i j}$ & Risk value \\
\hline$S_{11}$ & 0.6 \\
$S_{12}$ & 0.8 \\
$S_{13}$ & 0.2 \\
$S_{14}$ & 0.8 \\
$S_{15}$ & 0.7 \\
$S_{21}$ & 0.8 \\
$S_{22}$ & 0.8 \\
$S_{23}$ & 0.2 \\
$S_{24}$ & 0.7 \\
$S_{25}$ & 0.7 \\
\hline
\end{tabular}

Now, we incorporate the proposed ranking method and rank these two fuzzy probability numbers. Their ranking score for the failure modes are $\left(R\left(\pi_{2}\right)=0.010713\right), R\left(\pi_{4}\right)=$ $1.0185)$. Hence, $s_{2}$ has priority to perform corrective actions over $s_{1}$ because its fuzzy risk probability has a higher ranking score according to the ranking algorithm introduced in Section 4.

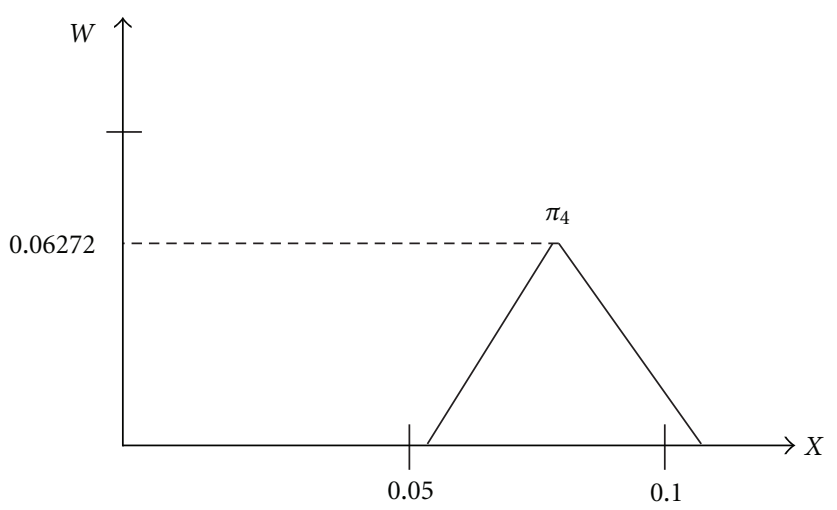

FIgURE 9: Fuzzy probability number $\pi_{4}$.

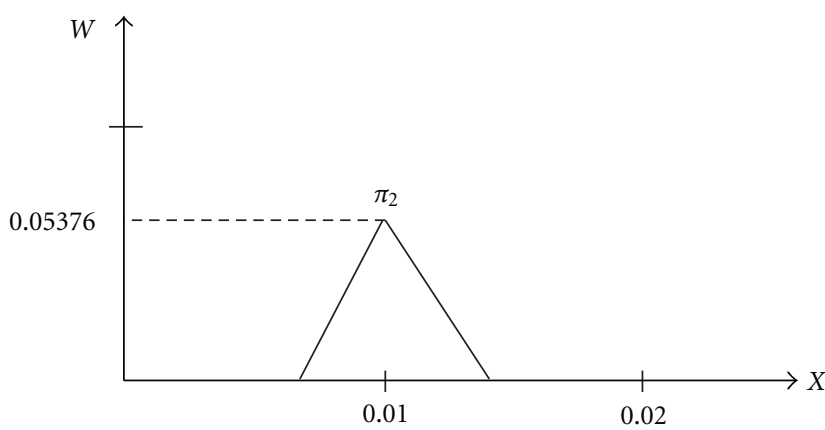

FIgURe 10: Fuzzy probability number $\pi_{2}$.

\section{Conclusions}

In this study we proposed a hybrid approach utilizing isomorphism between stochastic Petri Nets and Markov chains, and also a novel fuzzy ranking method. This approach is general and many different risk factors in the systems, which should be considered, are studied, for the first time. Another contribution of this paper is the application of fuzzy logic in determining steady state probabilities of systems and incorporating them in risk analysis.

The proposed methodology can be useful in most real life applications such as industrial systems; however, an important issue regarding this methodology is complexity. Although this method is quite efficient in dealing with small or medium sized Petri Nets but it would be hard to implement it on more complex nets. Therefore, presenting new approaches to improve efficiency of the prosed method in order to handle large scale problems can be an appropriate topic for future studies.

\section{References}

[1] M. Braglia, M. Frosolini, and R. Montanari, "Fuzzy critically assessment for failure modes and effect analysis," International Journal of Quality \& Reliability Management, vol. 20, no. 4, pp. 503-524, 2003.

[2] D. H. Stamatis, Failure Mode and Effect Analysis- FMEA From Theory to Execution, ASQC Quality Press, Milwaukee, Wis, USA, 1995. 
[3] K. Xu, L. C. Tang, M. Xie, S. L. Ho, and M. L. Zhu, "Fuzzy assessment of FMEA for engine systems," Reliability Engineering and System Safety, vol. 75, no. 1, pp. 17-29, 2002.

[4] C. L. Chang, C. C. Wei, and Y. H. Lee, "Failure mode and effects analysis using fuzzy method and grey theory," Kybernetes, vol. 28, no. 9, pp. 1072-1080, 1999.

[5] B. H. Lee, "Using Bayes belief networks in industrial FMEA modeling and analysis," in Proceedings of the International Symposium on Product Quality and Integrity, pp. 7-15, January 2001.

[6] M. Bevilacqua, "Monte Carlo simulation approach for a modified FMECA in a power plant," Quality and Reliability Engineering International, vol. 17, no. 9, pp. 3-13, 2000.

[7] A. D. Dominguez-Garcia, J. G. Kassakian, and J. E. Schindall, "Reliability evaluation of the power supply of an electrical power net for safety-relevant applications," Reliability Engineering and System Safety, vol. 91, no. 5, pp. 505-514, 2006.

[8] J. B. Bowles and C. E. Pelaez, "Application of fuzzy logic to reliability engineering," Proceedings of the IEEE, vol. 83, no. 3, pp. 435-449, 1995.

[9] K. J. Schmucker, Fuzzy Sets, Natural Language Computations, and Risk Analysis, Computer Science Press, Rockville, Md, USA, 1984.

[10] S. M. Chen and C. H. Wang, "Fuzzy risk analysis based on ranking fuzzy numbers using $\alpha$-cuts, belief features and signal/ noise ratios," Expert Systems with Applications, vol. 36, no. 3, pp. 5576-5581, 2009.

[11] S. M. Chen and J. H. Chen, "Fuzzy risk analysis based on ranking generalized fuzzy numbers with different heights and different spreads," Expert Systems with Applications, vol. 36, no. 3, pp. 6833-6842, 2009.

[12] S. M. Chen and J. H. Chen, "Fuzzy risk analysis based on similarity measures between interval-valued fuzzy numbers and interval-valued fuzzy number arithmetic operators," Expert Systems with Applications, vol. 36, no. 3, pp. 6309-6317, 2009.

[13] S. H. Wei and S. M. Chen, "A new approach for fuzzy risk analysis based on similarity measures of generalized fuzzy numbers," Expert Systems with Applications, vol. 36, no. 1, pp. 589-598, 2009.

[14] C. H. Cheng, "A new approach for ranking fuzzy numbers by distance method," Fuzzy Sets and Systems, vol. 95, no. 3, pp. 307-317, 1998.

[15] T. C. Chu and C. T. Tsao, "Ranking fuzzy numbers with an area between the centroid point and original point," Computers and Mathematics with Applications, vol. 43, no. 1-2, pp. 111-117, 2002.

[16] S. Murakami, S. Maeda, and S. Imamura, "Fuzzy decision analysis on the development of centralized regional energy control system," in Proceedings of IFAC Symposium on Fuzzy Informatics, Knowledge Representation and Decision Making, pp. 363-368, 1983.

[17] R. R. Yager, "Ranking fuzzy subsets over the unit interval," in Proceedings of 17th IEEE International Conference on Decision and Control, pp. 1436-1437, New York, NY, USA.

[18] S. J. Chen and S. M. Chen, "Fuzzy risk analysis basedon the ranking of generalized trapezoidal fuzzy numbers," Applied Intelligence, vol. 26, no. 1, pp. 1-11, 2007.

[19] L. W. Lee and S. M. Chen, "Fuzzy risk analysis based on fuzzy numbers with different shapes and different deviations," Expert Systems with Applications, vol. 34, no. 4, pp. 2763-2771, 2008.

[20] S. M. Chen and K. Sanguansat, "Analyzing fuzzy risk based on a new fuzzy ranking method between generalized fuzzy numbers," Expert Systems with Applications, vol. 38, no. 3, pp. 2163-2171, 2011.

[21] T. Murata, "Petri nets: properties, analysis and applications," Proceedings of the IEEE, vol. 77, no. 4, pp. 541-580, 1989.

[22] D. Vernez, D. Buchs, and G. Pierrehumbert, "Perspectives in the use of coloured Petri nets for risk analysis and accident modelling," Safety Science, vol. 41, no. 5, pp. 445-463, 2003.

[23] M. Zhou and K. Venkatesh, Modeling, Simulation And Control of Flexible Manufacturing Systems, A Petri Net Approach, World Scientific, Singapore, 1999.

[24] M. K. Molly, "Performance analysis using stochastic Petri Nets," IEEE Transactions on Computers, vol. 31, no. 9, pp. 913917, 1982.

[25] F. Bause and P. S. Kritzinger, Stochastic Petri Nets: An Introduction to The Theory, Vieweg, Braunschweig, Germany, 2nd edition, 2002.

[26] H. J. Zimmermann, Fuzzy Set Theory and Its Applications, Kluwer Academic, New York, NY, USA, 3rd edition, 1996.

[27] A. Doostparast Torshizi, Integrated risk analysis of systems based on Petri Nets analysis [Dissertation], Isfahan University of Technology, 2011.

[28] R. Viertl and D. Hareter, "Fuzzy information and stochastics," Iranian Journal of Fuzzy Systems, vol. 1, no. 1, pp. 39-52, 2004.

[29] F. Tüysüz and C. Kahraman, "Modeling a flexible manufacturing cell using stochastic Petri nets with fuzzy parameters," Expert Systems with Applications, vol. 37, no. 5, pp. 3910-3920, 2010.

[30] M. C. Zhou and K. Venkatesh, Modeling, Simulation and Control of Flexible Manufacturing Systems: A Petri Net Approach, World Scientific, Singapore, 1999. 

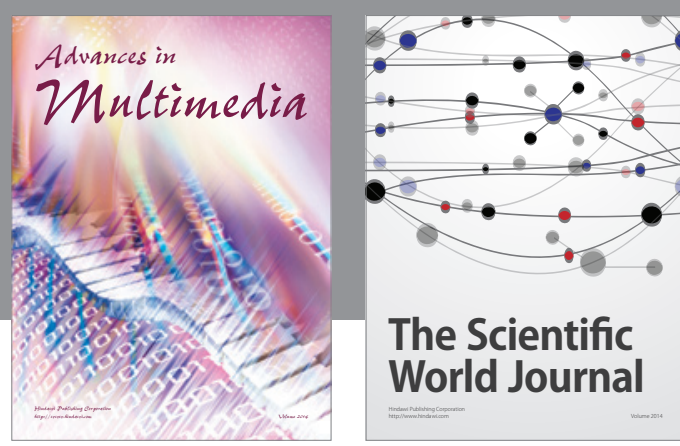

The Scientific World Journal
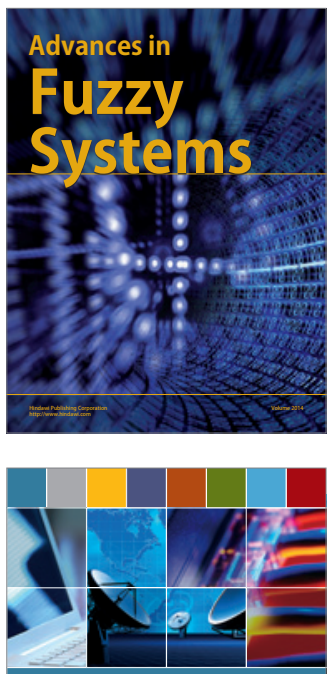

Computer Networks and Communications
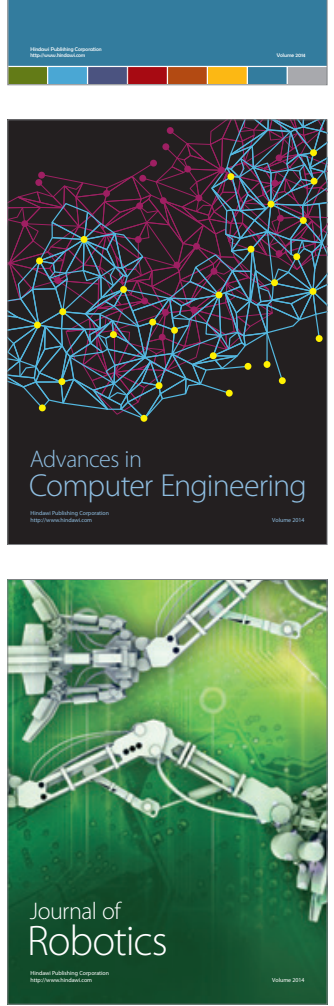
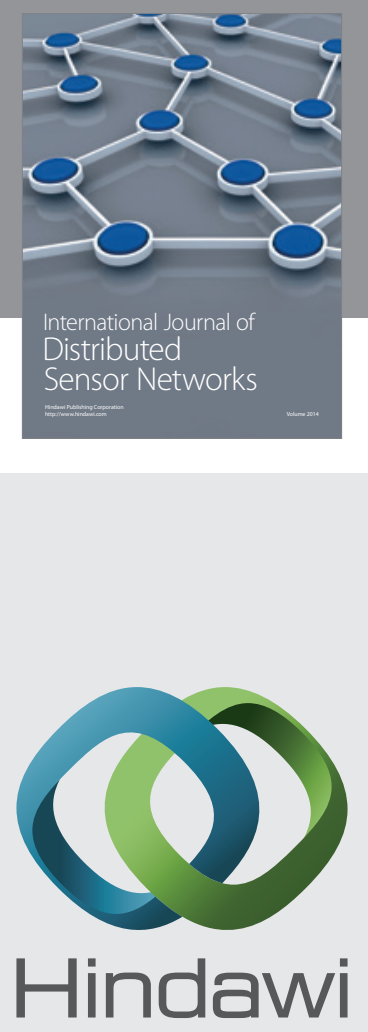

Submit your manuscripts at

http://www.hindawi.com
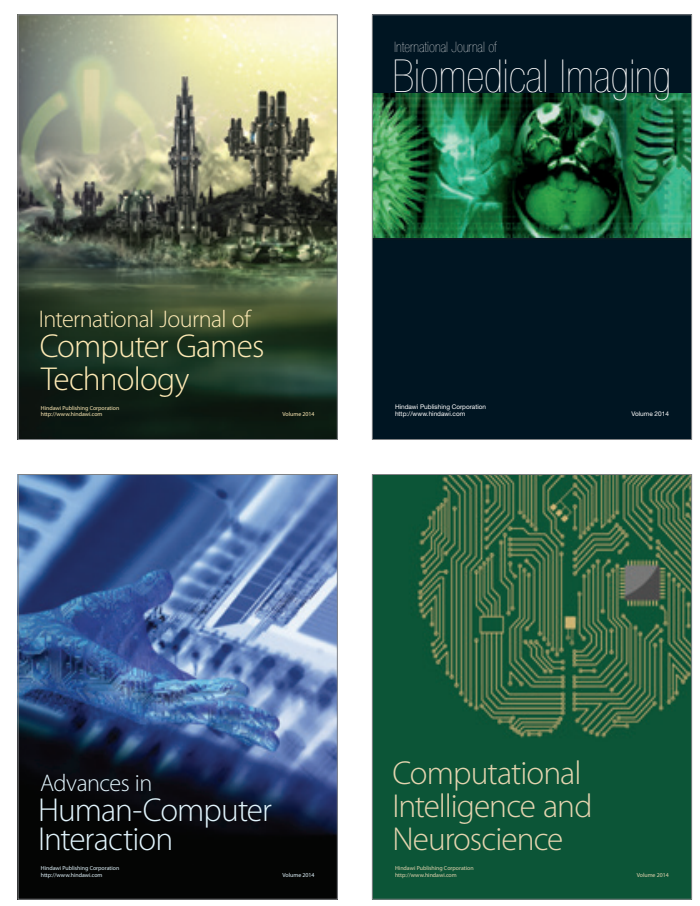
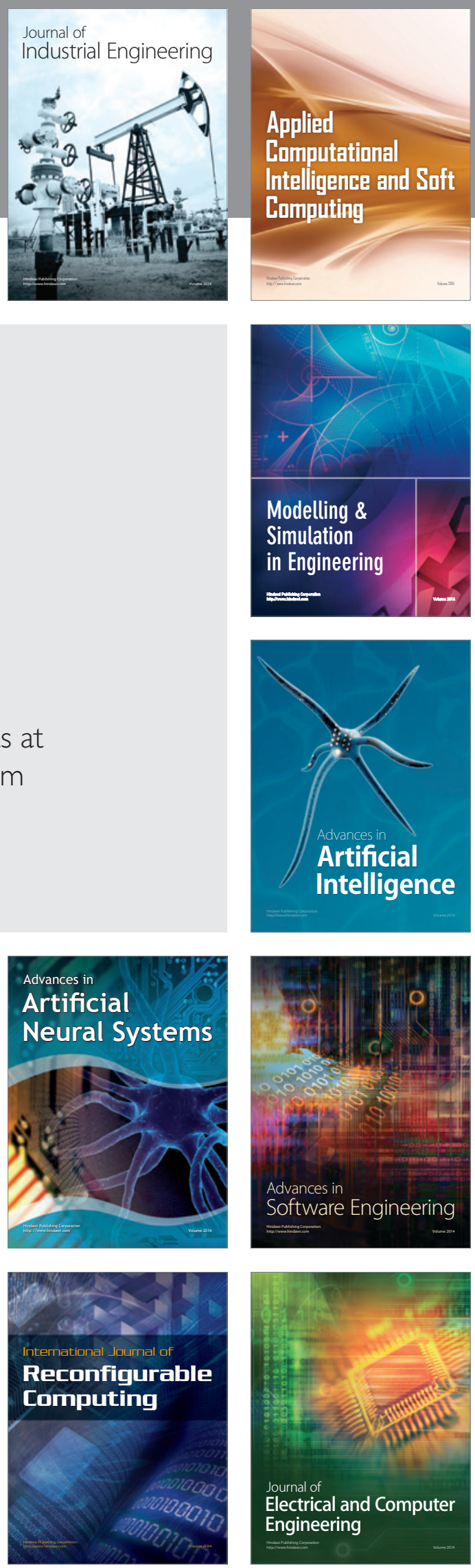